Kragujevac Journal of Mathematics

Volume 44(3) (2020), Pages 393-399.

\title{
ON CAPUTO FRACTIONAL DERIVATIVES VIA CONVEXITY
}

\author{
G. FARID ${ }^{1}$
}

\begin{abstract}
In this paper some estimations of Caputo fractional derivatives via convexity have been presented. By using convexity of any positive integer order differentiable function some novel results are given.
\end{abstract}

\section{INTRODUCTION}

Caputo fractional derivatives are defined as follows (see [1]).

Definition 1.1. Let $\alpha>0$ and $\alpha \notin\{1,2,3, \ldots\}, n=[\alpha]+1, f \in A C^{n}[a, b]$, the space of functions having $n t h$ derivatives absolutely continuous. The left-sided and right-sided Caputo fractional derivatives of order $\alpha$ are defined as follows:

$$
\left({ }^{C} D_{a+}^{\alpha} f\right)(x)=\frac{1}{\Gamma(n-\alpha)} \int_{a}^{x} \frac{f^{(n)}(t)}{(x-t)^{\alpha-n+1}} d t, \quad x>a
$$

and

$$
\left({ }^{C} D_{b-}^{\alpha} f\right)(x)=\frac{(-1)^{n}}{\Gamma(n-\alpha)} \int_{x}^{b} \frac{f^{(n)}(t)}{(t-x)^{\alpha-n+1}} d t, \quad x<b .
$$

If $\alpha=n \in\{1,2,3, \ldots\}$ and usual derivative $f^{(n)}(x)$ of order $n$ exists, then Caputo fractional derivative $\left({ }^{C} D_{a+}^{n} f\right)(x)$ coincides with $f^{(n)}(x)$ whereas $\left({ }^{C} D_{b-}^{n} f\right)(x)$ coincides with $f^{(n)}(x)$ with exactness to a constant multiplier $(-1)^{n}$. In particular we have

$$
\left({ }^{C} D_{a+}^{0} f\right)(x)=\left({ }^{C} D_{b-}^{0} f\right)(x)=f(x),
$$

where $n=1$ and $\alpha=0$.

Key words and phrases. Convex function, Caputo fractional derivatives.

2010 Mathematics Subject Classification. Primary: 26D15. Secondary: 26A51, 26A33.

DOI 10.46793/KgJMat2003.393F

Received: February 18, 2018.

Accepted: May 22, 2018. 
Since the inequalities always have been proved worthy in establishing the mathematical models and their solutions in almost all branches of applied sciences (see $[2,3]$ ). Especially the convexity takes very important role in the optimization theory. The aim of this paper is to introduce some fractional inequalities for the Caputo fractional derivatives via the convexity property of the functions which have derivatives of any integer order.

\section{Main Results}

First we give the following estimate of the sum of left and right handed Caputo fractional derivatives.

Theorem 2.1. Let $f: I \rightarrow \mathbb{R}$ be a real valued $n$-time differentiable function where $n$ is a positive integer. If $f^{(n)}$ is a positive convex function, then for $a, b \in I, a<b$ and $\alpha, \beta \geq 1$, the following inequality for Caputo fractional derivatives holds

$$
\begin{aligned}
& \Gamma(n-\alpha+1)\left({ }^{C} D_{a+}^{\alpha-1} f\right)(x)+\Gamma(n-\beta+1)\left({ }^{C} D_{b-}^{\beta-1} f\right)(x) \\
\leq & \frac{(x-a)^{n-\alpha+1} f^{(n)}(a)+(b-x)}{2} \\
& +f^{(n)}(x)\left[\frac{(x-a)^{n-\alpha+1}+(b-x)^{n-\beta+1}}{2}\right] .
\end{aligned}
$$

Proof. Let us consider the function $f$ on the interval $[a, x], x \in[a, b]$. For $t \in[a, x]$, the following inequality holds

$$
(x-t)^{n-\alpha} \leq(x-a)^{n-\alpha} .
$$

Since $f^{(n)}$ is convex therefore for $t \in[a, x]$ we have

$$
f^{(n)}(t) \leq \frac{x-t}{x-a} f^{(n)}(a)+\frac{t-a}{x-a} f^{(n)}(x) .
$$

Multiplying inequalities (2.3) and (2.2), then integrating with respect to $t$ over $[a, x]$ we have

$$
\int_{a}^{x}(x-t)^{n-\alpha} f^{(n)}(t) d t \leq \frac{(x-a)^{n-\alpha}}{x-a}\left[f^{(n)}(a) \int_{a}^{x}(x-t) d t+f^{(n)}(x) \int_{a}^{x}(t-a) d t\right],
$$

$$
\Gamma(n-\alpha+1)\left({ }^{C} D_{a+}^{\alpha-1} f\right)(x) \leq \frac{(x-a)^{n-\alpha+1}}{2}\left[f^{(n)}(a)+f^{(n)}(x)\right] .
$$

Now we consider function $f$ on the interval $[x, b], x \in[a, b]$. For $t \in[x, b]$ the following inequality holds

$$
(t-x)^{n-\beta} \leq(b-x)^{n-\beta} .
$$

Since $f^{(n)}$ is convex on $[a, b]$, therefore, for $t \in[x, b]$ we have

$$
f^{(n)}(t) \leq \frac{t-x}{b-x} f^{(n)}(b)+\frac{b-t}{b-x} f^{(n)}(x) .
$$


Multiplying inequalities (2.5) and (2.6), then integrating with respect to $t$ over $[x, b]$ we have

$$
\begin{aligned}
& \int_{x}^{b}(t-x)^{n-\beta} f^{(n)}(t) d t \leq \frac{(b-x)^{n-\beta}}{b-x}\left[f^{(n)}(b) \int_{x}^{b}(t-x) d t+f^{(n)}(x) \int_{x}^{b}(b-t) d t\right], \\
& \text { 7) } \quad \Gamma(n-\beta+1)\left({ }^{C} D_{b-}^{\beta-1} f\right)(x) \leq \frac{(b-x)^{n-\beta+1}}{2}\left[f^{(n)}(b)+f^{(n)}(x)\right] .
\end{aligned}
$$

Adding (2.4) and (2.7) we get the required inequality in (2.1).

It is nice to see that the following implication holds.

Corollary 2.1. By setting $\alpha=\beta$ in (2.1) we get the following fractional integral inequality

$$
\begin{aligned}
& \Gamma(n-\alpha+1)\left(\left({ }^{C} D_{a+}^{\alpha-1} f\right)(x)+\left({ }^{C} D_{b-}^{\alpha-1} f\right)(x)\right) \\
\leq & \frac{(x-a)^{n-\alpha+1} f^{(n)}(a)+(b-x)^{n-\alpha+1} f^{(n)}(b)}{2}+f^{(n)}(x)\left[\frac{(x-a)^{n-\alpha+1}+(b-x)^{n-\alpha+1}}{2}\right] .
\end{aligned}
$$

Now we give the next result stated in the following theorem.

Theorem 2.2. Let $f: I \rightarrow \mathbb{R}$ be a real valued $n$-time differentiable function, where $n$ is a positive integer. If $\left|f^{(n+1)}\right|$ is convex function, then for $a, b \in I a<b$ and $\alpha, \beta>0$, the following inequality for Caputo fractional derivatives holds

$$
\begin{aligned}
& \mid \Gamma(n-\alpha+1)\left({ }^{C} D_{a+}^{\alpha} f\right)(x)+\Gamma(n-\beta+1)\left({ }^{C} D_{b-}^{\beta} f\right)(x) \\
& -\left((x-a)^{n-\alpha} f^{(n)}(a)+(b-x)^{n-\beta} f^{(n)}(b)\right) \mid \\
\leq & \frac{(x-a)^{\alpha+1}\left|f^{(n+1)}(a)\right|+(b-x)^{\beta+1}\left|f^{(n+1)}(b)\right|}{2} \\
& +\frac{\left|f^{(n+1)}(x)\right|\left((x-a)^{\alpha+1}+(b-x)^{\beta+1}\right)}{2} .
\end{aligned}
$$

Proof. Since $\left|f^{(n+1)}\right|$ is convex, therefore, for $t \in[a, x]$ we have

$$
\left|f^{(n+1)}(t)\right| \leq \frac{x-t}{x-a}\left|f^{(n+1)}(a)\right|+\frac{t-a}{x-a}\left|f^{(n+1)}(x)\right|
$$

from which we can write

$$
\begin{aligned}
-\left(\frac{x-t}{x-a}\left|f^{(n+1)}(a)\right|+\frac{t-a}{x-a}\left|f^{(n+1)}(x)\right|\right) & \leq f^{(n+1)}(t) \\
& \leq \frac{x-t}{x-a}\left|f^{(n+1)}(a)\right|+\frac{t-a}{x-a}\left|f^{(n+1)}(x)\right| .
\end{aligned}
$$

We consider the second inequality of inequality (2.9)

$$
f^{(n+1)}(t) \leq \frac{x-t}{x-a}\left|f^{(n+1)}(a)\right|+\frac{t-a}{x-a}\left|f^{(n+1)}(x)\right| .
$$


Now for $\alpha>0$ we have

$$
(x-t)^{n-\alpha} \leq(x-a)^{n-\alpha}, t \in[a, x] .
$$

The product of last two inequalities give

$$
(x-t)^{n-\alpha} f^{(n+1)}(t) \leq(x-a)^{n-\alpha-1}\left((x-t)\left|f^{(n+1)}(a)\right|+(t-a)\left|f^{(n+1)}(x)\right|\right) .
$$

Integrating with respect to $t$ over $[a, x]$ we have

$$
\begin{aligned}
& \int_{a}^{x}(x-t)^{n-\alpha} f^{(n+1)}(t) d t \\
\leq & (x-a)^{n-\alpha-1}\left(\left|f^{(n+1)}(a)\right| \int_{a}^{x}(x-t) d t+\left|f^{(n+1)}(x)\right| \int_{a}^{x}(t-a) d t\right) \\
= & (x-a)^{n-\alpha+1}\left(\frac{\left|f^{(n+1)}(a)\right|+\left|f^{(n+1)}(x)\right|}{2}\right)
\end{aligned}
$$

and

$$
\begin{aligned}
\int_{a}^{x}(x-t)^{n-\alpha} f^{(n+1)}(t) d t & =\left.f^{(n)}(t)(x-t)^{n-\alpha}\right|_{a} ^{x}+(n-\alpha) \int_{a}^{x}(x-t)^{n-\alpha-1} f^{(n)}(t) d t \\
& =-f^{(n)}(a)(x-a)^{n-\alpha}+\Gamma(n-\alpha+1)\left({ }^{C} D_{a+}^{\alpha} f\right)(x) .
\end{aligned}
$$

Therefore, (2.11) takes the form

$$
\begin{aligned}
& \Gamma(n-\alpha+1)\left({ }^{C} D_{a+}^{\alpha} f\right)(x)-f^{(n)}(a)(x-a)^{n-\alpha} \\
\leq & (x-a)^{n-\alpha+1}\left(\frac{\left|f^{(n+1)}(a)\right|+\left|f^{(n+1)}(x)\right|}{2}\right) .
\end{aligned}
$$

If one consider from (2.9) the first inequality and proceed as we did for the second inequality, then following inequality can be obtained

$$
\begin{aligned}
& f^{(n)}(a)(x-a)^{n-\alpha}-\Gamma(n-\alpha+1)\left({ }^{C} D_{a+}^{\alpha} f\right)(x) \\
\leq & (x-a)^{n-\alpha+1}\left(\frac{\left|f^{(n+1)}(a)\right|+\left|f^{(n+1)}(x)\right|}{2}\right) .
\end{aligned}
$$

From (2.12) and (2.13) we get

$$
\begin{aligned}
& \left|\Gamma(n-\alpha+1)\left({ }^{C} D_{a+}^{\alpha} f\right)(x)-f^{(n)}(a)(x-a)^{n-\alpha}\right| \\
\leq & (x-a)^{n-\alpha+1}\left(\frac{\left|f^{(n+1)}(a)\right|+\left|f^{(n+1)}(x)\right|}{2}\right) .
\end{aligned}
$$

On the other hand for $t \in[x, b]$ using convexity of $\left|f^{(n+1)}\right|$ we have

$$
\left|f^{(n+1)}(t)\right| \leq \frac{t-x}{b-x}\left|f^{(n+1)}(b)\right|+\frac{b-t}{b-x}\left|f^{(n+1)}(x)\right| .
$$

Also for $t \in[x, b]$ and $\beta>0$ we have

$$
(t-x)^{n-\beta} \leq(b-x)^{n-\beta} .
$$


By adopting the same treatment as we have done for (2.9) and (2.10) one can obtain from (2.15) and (2.16) the following inequality

$$
\begin{aligned}
& \left|\Gamma(n-\beta+1)\left({ }^{C} D_{b-}^{\beta} f\right)(x)-f^{(n)}(b)(b-x)^{n-\beta}\right| \\
\leq & (b-x)^{n-\beta+1}\left(\frac{\left|f^{(n+1)}(b)\right|+\left|f^{(n+1)}(x)\right|}{2}\right) .
\end{aligned}
$$

By combining the inequalities (2.14) and (2.17) via triangular inequality we get the required inequality.

It is interesting to see the following inequalities as a special case.

Corollary 2.2. By setting $\alpha=\beta$ in (2.8) we get the following fractional integral inequality

$$
\begin{gathered}
\mid \Gamma(n-\alpha+1)\left[\left({ }^{C} D_{a+}^{\alpha} f\right)(x)+\left({ }^{C} D_{b-}^{\alpha} f\right)(x)\right] \\
-\left((x-a)^{n-\alpha} f^{(n)}(a)+(b-x)^{n-\alpha} f^{(n)}(b)\right) \mid \\
\leq \frac{(x-a)^{n-\alpha+1}\left|f^{(n+1)}(a)\right|+(b-x)^{n-\alpha+1}\left|f^{(n+1)}(b)\right|}{2} \\
+\frac{\left|f^{(n+1)}(x)\right|\left((x-a)^{n-\alpha+1}+(b-x)^{n-\alpha+1}\right)}{2} .
\end{gathered}
$$

Before going to the next theorem we observe the following result.

Lemma 2.1. Let $f:[a, b] \rightarrow \mathbb{R}$, be a convex function. If $f$ is symmetric about $\frac{a+b}{2}$, then the following inequality holds

$$
f\left(\frac{a+b}{2}\right) \leq f(x), \quad x \in[a, b] .
$$

Proof. We have

$$
\frac{a+b}{2}=\frac{1}{2}\left(\frac{x-a}{b-a} b+\frac{b-x}{b-a} x\right)+\frac{1}{2}\left(\frac{x-a}{b-a} a+\frac{b-x}{b-a} b\right) .
$$

Since $f$ is convex, therefore we have

$$
\begin{aligned}
f\left(\frac{a+b}{2}\right) & \leq \frac{1}{2}\left(f\left(\frac{x-a}{b-a} b+\frac{b-x}{b-a} x\right)+f\left(\frac{x-a}{b-a} a+\frac{b-x}{b-a} b\right)\right) \\
& =\frac{1}{2}(f(x)+f(a+b-x)) .
\end{aligned}
$$

Also $f$ is symmetric about $\frac{a+b}{2}$, therefore, we have $f(a+b-x)=f(x)$ and inequality in (2.18) holds.

Theorem 2.3. Let $f: I \rightarrow \mathbb{R}$ be a real valued $n$-time differentiable function where $n$ is a positive integer. If $f^{(n)}$ is a positive convex and symmetric about $\frac{a+b}{2}$, then for 
$a, b \in I, a<b$ and $\alpha, \beta \geq 1$, the following inequality for Caputo fractional derivatives holds

$$
\begin{aligned}
& \frac{1}{2}\left(\frac{1}{n-\alpha+1}+\frac{1}{n-\beta+1}\right) f^{(n)}\left(\frac{a+b}{2}\right) \\
\leq & \frac{\Gamma(n-\beta+1)\left({ }^{C} D_{b-}^{\beta-1} f\right)(a)}{2(b-a)^{n-\beta+1}}+\frac{\Gamma(n-\alpha+1)\left({ }^{C} D_{a+}^{\alpha-1} f\right)(b)}{2(b-a)^{n-\alpha+1}} \\
\leq & \frac{f^{(n)}(a)+f^{(n)}(b)}{2} .
\end{aligned}
$$

Proof. For $x \in[a, b]$ we have

$$
(x-a)^{n-\beta} \leq(b-a)^{n-\beta} .
$$

Also $f$ is convex function we have

$$
f^{(n)}(x) \leq \frac{x-a}{b-a} f^{(n)}(b)+\frac{b-x}{b-a} f^{(n)}(a) .
$$

Multiplying (2.20) and (2.21) and then integrating with respect to $x$ over $[a, b]$ we have

$$
\int_{a}^{b}(x-a)^{n-\beta} f^{(n)}(x) d x \leq \frac{(b-a)^{n-\beta}}{b-a}\left(\int_{a}^{b}\left(f^{(n)}(b)(x-a)+f^{(n)}(a)(b-x)\right) d x\right) .
$$

From which we have

$$
\frac{\Gamma(n-\beta+1)\left({ }^{C} D_{b-}^{\beta-1} f\right)(a)}{(b-a)^{n-\beta+1}} \leq \frac{f^{(n)}(a)+f^{(n)}(b)}{2} .
$$

On the other hand for $x \in[a, b]$ we have

$$
(b-x)^{n-\alpha} \leq(b-a)^{n-\alpha} .
$$

Multiplying (2.21) and (2.23) and then integrating with respect to $x$ over $[a, b]$ we get

$$
\int_{a}^{b}(b-x)^{n-\alpha} f^{(n)}(x) d x \leq(b-a)^{n-\alpha+1} \frac{f^{(n)}(a)+f^{(n)}(b)}{2} .
$$

From which we have

$$
\frac{\Gamma(n-\alpha+1)\left({ }^{C} D_{a+}^{\alpha-1} f\right)(b)}{(b-a)^{n-\alpha+1}} \leq \frac{f^{(n)}(a)+f^{(n)}(b)}{2} .
$$

Adding (2.22) and (2.24) we get the second inequality

$$
\frac{\Gamma(n-\beta+1)\left({ }^{C} D_{b-}^{\beta-1} f\right)(a)}{2(b-a)^{n-\beta+1}}+\frac{\Gamma(n-\alpha+1)\left({ }^{C} D_{a+}^{\alpha-1} f\right)(b)}{2(b-a)^{n-\alpha+1}} \leq \frac{f^{(n)}(a)+f^{(n)}(b)}{2} .
$$

Since $f^{(n)}$ is convex and symmetric about $\frac{a+b}{2}$ using Lemma 2.1 we have

$$
f^{(n)}\left(\frac{a+b}{2}\right) \leq f^{(n)}(x), \quad x \in[a, b] .
$$


Multiplying with $(x-a)^{n-\beta}$ on both sides and then integrating over $[a, b]$ we have

$$
f^{(n)}\left(\frac{a+b}{2}\right) \int_{a}^{b}(x-a)^{n-\beta} d x \leq \int_{a}^{b}(x-a)^{n-\beta} f^{(n)}(x) d x .
$$

By definition of Caputo fractional derivatives one can has

$$
f^{(n)}\left(\frac{a+b}{2}\right) \frac{1}{2(n-\beta+1)} \leq \frac{\Gamma(n-\beta+1)\left({ }^{C} D_{b-}^{\beta-1} f\right)(a)}{2(b-a)^{n-\beta+1}} .
$$

Multiplying $(2.25)$ with $(b-x)^{n-\alpha}$, then integrating over $[a, b]$ one can get

$$
f^{(n)}\left(\frac{a+b}{2}\right) \frac{1}{2(n-\alpha+1)} \leq \frac{\Gamma(n-\alpha+1)\left({ }^{C} D_{a+}^{\alpha-1} f\right)(b)}{2(b-a)^{n-\alpha+1}} .
$$

Adding (2.26) and (2.27) we get the first inequality.

Corollary 2.3. If we put $\alpha=\beta$ in (2.19), then we get

$$
\begin{aligned}
f^{(n)}\left(\frac{a+b}{2}\right) \frac{1}{\alpha+1} & \leq \frac{\Gamma(n-\alpha+1)}{2(b-a)^{\alpha+1}}\left(\left({ }^{C} D_{b-}^{\alpha+1} f\right)(a)+\left({ }^{C} D_{a+}^{\alpha+1} f\right)(b)\right) \\
& \leq \frac{f^{(n)}(a)+f^{(n)}(b)}{2}
\end{aligned}
$$

Acknowledgements. This research work is supported by Higher Education Commission of Pakistan under NRPU 2016, Project No. 5421.

\section{REFERENCES}

[1] A. A. Kilbas, H. M. Srivastava and J. J. Trujillo, Theory and Applications of Fractional Differential Equations, North-Holland Mathematics Studies 204, Elsevier, New York, London, 2006.

[2] M. Lazarević, Advanced Topics on Applications of Fractional Calculus on Control Problems, System Stability and Modeling, WSEAS Press, Belgrade, Serbia, 2012.

[3] K. Oldham and J. Spanier, The Fractional Calculus Theory and Applications of Differentiation and Integration to Arbitrary Order, Academic Press, New York, London, 1974.

${ }^{1}$ Department of Mathematics,

COMSATS University Islamabad, Attock Campus, Pakistan

Email address: faridphdsms@hotmail.com, ghlmfarid@cuiatk.edu.pk 